\title{
The effects of yeast metabolites on the rheological behaviour of the dough matrix in fermented wheat flour dough
}

\author{
Mathieu Meerts ${ }^{\mathrm{a}}$ \\ Ana Ramirez Cervera ${ }^{a}$ \\ Nore Struyf ${ }^{b}$ \\ Ruth Cardinaels ${ }^{\mathrm{a}, \mathrm{c}} \quad$ Christophe M. Courtin ${ }^{\mathrm{b}} \quad$ Paula Moldenaers $^{\mathrm{a}}$ \\ a Soft Matter, Rheology and Technology, Department of Chemical Engineering, KU Leuven, Celestij- \\ nenlaan 200F, Box 2424, BE 3001 Leuven, Belgium \\ b Laboratory of Food Chemistry and Biochemistry \& Leuven Food Science and Nutrition Research \\ Center (LFoRCe), Department of Microbial and Molecular Systems, KU Leuven, Kasteelpark Aren- \\ berg 22, Box 2463, BE 3001 Leuven, Belgium \\ c Polymer Technology, Department of Mechanical Engineering, TU Eindhoven, Box 513, 5600 MB \\ Eindhoven, The Netherlands
}

Corresponding author: mathieu.meerts@cit.kuleuven.be 


\begin{abstract}
In this study, the rheological impact of the main yeast metabolites besides $\mathrm{CO}_{2}$ was investigated. By adding these metabolites to unfermented dough at the concentrations observed in fermented dough, the associated rheological changes could be examined with fundamental rheological techniques (oscillatory and uniaxial extensional tests). Glycerol had a softening effect on dough similar to water. Ethanol altered the configuration of the gluten network, resulting in a decrease in the dough's extensional viscosity and extensibility. The stiffness and extensional viscosity were also reduced by succinic acid and glutathione.

Subsequently, the impact of these metabolites on the rheology of dough was also investigated in situ by studying the rheological changes in the dough matrix following fermentation. Compared to unfermented dough, the fermented dough matrix exhibited reduced extensibility and a lower maximum extensional viscosity. The storage modulus also decreased, but only at low frequencies. The observed changes can partially be accounted for by the yeast metabolites, yet it is clear that the rheological behaviour of the fermented dough matrix does not merely resemble a superposition of the rheological changes associated with the main yeast metabolites. The differences may reflect the time-dependent accumulation of metabolites in the expanding gluten network during fermentation.
\end{abstract}

Keywords Dough rheology; Yeast fermentation; Ethanol; Succinic acid

\title{
1 Introduction
}

Baker's yeast is the principal leavening agent in the breadmaking process (Jayaram et al., 2013). During fermentation, yeast cells produce $\mathrm{CO}_{2}$, which is retained within the dough by a complex matrix consisting of a viscoelastic gluten protein-starch network. The functionality of yeast in breadmaking is, however, not limited to gas production, as yeast produces several other metabolites that may affect the final product quality. Recently, Courtin and co-workers accurately determined the nature and relative amounts of the metabolites produced by yeast during dough fermentation (Jayaram et al., 2013). Besides the primary metabolites $\mathrm{CO}_{2}$ and ethanol, also significant amounts of secondary metabolites, such as succinic acid, acetic acid and glycerol, were detected in fermented dough. Contrary to common belief, the drop in $\mathrm{pH}$ associated with fermentation is not only caused by the dissolution of $\mathrm{CO}_{2}$ in the aqueous dough phase, but mainly stems from the accumulation of succinic acid (Jayaram et al., 2013). Furthermore, by means of empirical rheological techniques (Kieffer extensibility rig, Chopin Alveograph), Courtin and co-workers established that these metabolites had a clear impact on the tensile strength and the extensibility of (unfermented) dough (Jayaram et al., 2014a,b; Aslankoohi et al., 2015). In addition, Verheyen et al. (2015) found that glutathione, which may end up in the dough matrix through leakage from dead yeast cells, greatly softens the dough, as evidenced by a substantial decrease in mixing stability and a sharp 
decline in the complex modulus $G^{*}$.

The rheological properties of dough are known to be intrinsically linked to the final quality of the baked product. Despite the obvious importance of the fermentation step in the breadmaking process, the number of (fundamental) rheological studies dealing with fermented dough is surprisingly limited. The reason is that in vivo yeast activity leads to a very complex system that is difficult to characterise both from a microstructural and rheological point of view, as metabolites are produced in situ and their concentrations vary with time. To accurately probe the rheological behaviour of fermented dough, it is necessary to stop the fermentation, preferably by non-invasive methods. This is, however, far from straightforward to achieve. Newberry et al. (2002) used a freeze-two stage thawing procedure to inactivate the yeast, which was later adopted by Salvador et al. (2006) and Connelly and McIntier (2008). Newberry et al. (2002) did not observe any changes in the dynamic moduli as a result of fermentation, whereas Salvador et al. (2006) and Connelly and McIntier (2008) did report a strong decrease in both $G^{\prime}$ and $G^{\prime \prime}$. The thermal inactivation approach proved successful in halting the yeast activity after any desired fermentation time, yet the freezing and subsequent thawing of dough obviously also affected its rheological behaviour. Other rheological studies on fermented dough chose to not inactivate the yeast (e.g. Kilborn and Preston (1982), Wehrle and Arendt (1998)). It is, however, important to realise that the ongoing fermentation might confound the rheological data (Newberry et al., 2002). The fermented dough systems exhibited a strong decline in tensile strength and extensibility (Kilborn and Preston, 1982) and a substantial decrease in complex viscosity (Wehrle and Arendt, 1998), which can be attributed to the significant changes in dough density (Newberry et al., 2002).

The present study aims to assess the rheological impact of the main yeast metabolites on wheat flour dough, by adding these metabolites to unfermented dough in their respective concentrations. A second objective of this work is to study the rheological behaviour of the dough matrix in fermented dough, which will allow us to determine whether the main yeast metabolites can explain the differences in the rheological behaviour of fermented dough as compared to unfermented dough. The $\mathrm{CO}_{2}$ gas bubbles are known to have a substantial softening effect on dough (Verheyen et al., 2014). In this study we are, however, interested in the rheological changes associated with the dough matrix rather than with $\mathrm{CO}_{2}$ production, as the rheology of the dough matrix determines the ability of the dough to expand and subsequently retain its shape during proofing and baking. For that reason, gas bubbles are removed from the fermented dough by (repetitive) sheeting. To circumvent the issue of yeast inactivation when studying the rheology of fermented dough, we let the yeast ferment until its primary sugar sources are depleted, after which its activity drops significantly. The effects of the yeast metabolites on dough rheology are examined with fundamental rather than empirical rheological techniques. Linear behaviour in shear is investigated by means of small amplitude oscillatory shear (SAOS) tests, while for the rheological characterisation in uniaxial extensional flow, we use an Extensional Viscosity Fixture (EVF) mounted on a rotational rheometer. 


\section{Materials and methods}

\subsection{Dough preparation}

The flour used in this study (commercial Bison flour, no additives) originated from Dossche Mills (Deinze, Belgium). The moisture content of the flour was $12.7 \pm 0.05$ wt\%, according to AACC method 44-19.01 (AACCI, 2000). The protein content ( $\mathrm{N} \mathrm{x}$ 5.7 ) of the flour was measured with an automated Dumas protein analysis system (EAS, VarioMax N/CN, Elt, Gouda, The Netherlands) following an adaptation of the AOAC method 990.03 (AOACI, 1995), and amounted to $12.4 \pm 1.0 \mathrm{wt} \%$ (on a dry matter basis). All analyses were done in triplicate.

The optimal water absorption and mixing time were determined with a Farinograph (Brabender, Duisburg, Germany) and a Mixograph (National Manufacturing, Lincoln, USA) in accordance with AACC methods 54-40.02 and 54-21.02, respectively (AACCI, 2000). Dough samples consisted of $10 \mathrm{~g}$ flour (on $14 \mathrm{wt} \%$ moisture base), $1.5 \mathrm{wt} \%$ sodium chloride, $6 \mathrm{wt} \%$ sucrose, and $5.4 \mathrm{ml}$ of water (AACC method 10-10.03). To elucidate the effects of the yeast metabolites ethanol, succinic acid, glycerol and glutathione on dough rheology, representative amounts of these metabolites were also added to unfermented dough. Ethanol was obtained from Fisher Scientific (Loughborough, UK), succinic acid and glutathione from Sigma-Aldrich (Overijse, Belgium) and glycerol from Thermofisher Acros Organics (Geel, Belgium). The fermented dough samples additionally contained $5.3 \mathrm{wt} \%$ of compressed baker's yeast (Algist Bruggeman, Ghent, Belgium), which represents an upper limit for the yeast concentrations used in industry (Mondal and Datta, 2008). All ingredients were mixed in a $10 \mathrm{~g}$ pin bowl mixer (National Manufacturing) for 3 min $30 \mathrm{~s}$ to reach the optimal consistency. After mixing, the dough samples were shaped with a pasta machine to obtain a quasi-cylindrical disc with a height of ca. 4 $\mathrm{mm}$ and a diameter of about $40 \mathrm{~mm}$.

\subsection{Determination of the yeast activity}

The volume of gas produced in dough during fermentation can be recorded with a Risograph instrument (National Manufacturing). Risograph measurements on fermenting Bison dough indicated that after 6 hours the fermentation rate at $30^{\circ} \mathrm{C}$ dropped to almost zero, due to depletion of the readily available fermentable sugars. Hence, after being shaped with the pasta machine the fermenting dough samples were covered by a small metal bowl and rested for 6 hours in a climate chamber (HPP 749, Memmert, Schwabach, Germany) held at $30^{\circ} \mathrm{C}$ and a relative humidity of $75 \%$, in order to avoid any confounding of the rheological data by ongoing yeast activity. After this fermentation period of 6 hours, the fermented dough samples were sheeted again with the pasta machine to remove the entrapped $\mathrm{CO}_{2}$ from the dough matrix (Rezaei et al., 2016), 
before being loaded in the rheometer. Following this second sheeting step, no more gas bubbles could be detected with the naked eye. Sheeting the dough samples immediately afterwards for a third time did not change their dynamic rheological response (results not shown), implying that all gas bubbles that could be removed by sheeting, were already removed in the second sheeting step. To allow a proper comparison, the unfermented dough samples (with or without added yeast metabolites) were given exactly the same rheological pretreatment as the fermented dough.

\subsection{Determination of the metabolite concentrations}

The metabolite concentrations (except for glutathione) in fermented dough were determined with high performance liquid chromatography (HPLC). After a fermentation period of 6 hours in the Risograph, the dough samples were weighed and an amount of deionised water equal to twice the dough's weight was added. Subsequently, the dough-water mixture was blended in a commercial blender 8011E (Waring Products, Torrington, USA) for 30 seconds, and then centrifuged in an Eppendorf centrifuge 5415D (Eppendorf AG, Hamburg, Germany) for 3 minutes. The supernatant was filtered by means of a 0.22- $\mu \mathrm{m}$, polyethersulfone membrane (Millex-GP, Millipore, Carrightwohill, Ireland) and stored at $-18{ }^{\circ} \mathrm{C}$ for further analysis. Analyses were performed in triplicate. Ethanol, succinic acid, glycerol, acetic acid and lactic acid were separated and quantified by an LC-20AT modular HPLC system (Shimadzu, Kyoto, Japan) using an ion exclusion column ROA-Organic acids (Phenomenex, Torrance, USA) and a refractive index detection system (Shimadzu, RID-10A detector). The column was operated at a temperature of $60^{\circ} \mathrm{C}$ and with $2.50 \mathrm{mM} \mathrm{H}_{2} \mathrm{SO}_{4}$ as eluent at a flow rate of 0.60 $\mathrm{ml} / \mathrm{min}$. Methanol was used as a cleaning solvent for the injection syringe and could be detected in all HPLC profiles.

The $\mathrm{pH}$ of unfermented dough supplemented with succinic acid was determined with a pH probe (HI 9126, Hanna Instruments, Temse, Belgium), which was placed directly in the sample immediately after the mixing step. Three samples were prepared for each condition, and each sample was measured twice.

\subsection{Rheological methods}

Small amplitude oscillatory shear (SAOS) tests were performed at $25^{\circ} \mathrm{C}$ on a stresscontrolled MCR501 rheometer (Anton Paar, Graz, Austria) equipped with a $40 \mathrm{~mm}$ parallel plate geometry coated with sandpaper and covered with a solvent trap. After loading in the rheometer, the dough sample was allowed to rest for 5 min to allow the remaining stresses to relax (see section 3.2.1). Subsequently, frequency sweeps were obtained in the linear region. All dynamic measurements were performed in triplicate on separately prepared batches of dough (with the average values being shown). Good reproducibility was obtained, both for unfermented and fermented dough, with relative standard deviations being always less than $11 \%$. To determine whether the observed 
differences between dough samples prepared with different metabolites were statistically significant, the two-tailed unpaired Welch's t-test was used.

Dough behaviour in extension was studied at ambient temperature (ca. $19-22{ }^{\circ} \mathrm{C}$ ) by means of the Extensional Viscosity Fixture (EVF) mounted on a strain-controlled ARES-G2 rheometer (TA Instruments, New Castle, USA). The EVF setup consists of two drums to which the dough strand can be attached. Extension at a constant rate is obtained as one drum remains stationary and the other moves in a circular orbit around it whilst also rotating around its own axis. In a previous study (Meerts et al., 2017), we determined that the dough samples should have a height-to-thickness ratio $\left(H_{0} / B_{0}\right)$ close to unity to exhibit a homogeneous uniaxial extension in the EVF. Hence, the sample height (in the vertical direction) and the sample thickness were set to $4 \mathrm{~mm}$, whereas the initial sample length $L_{0}$ (in the horizontal direction) amounted to $12.5 \mathrm{~mm}$. A suitable measure for the deformation is the Hencky strain $\epsilon(t)[-]$ :

$$
\epsilon(t)=\ln \left(\frac{L(t)}{L_{0}}\right)
$$

in which $L_{0}$ represents the initial length of the dough strand as mentioned previously, and $L(t)$ is the actual length during extension. The transient extensional viscosity $\eta_{e}^{+}(\epsilon)$ $[\mathrm{Pa} \mathrm{s}]$ is defined as:

$$
\eta_{e}^{+}(\epsilon)=\frac{\sigma_{11}(\epsilon)}{\dot{\epsilon}}
$$

In this expression $\sigma_{11}(\epsilon)$ corresponds to the longitudinal stress $[\mathrm{Pa}]$ calculated from the torque registered by the transducer of the rheometer. Following the 6-hour stay in the climate chamber and the second sheeting action, the dough samples were rested for an additional 15 min under a metal bowl and finally loaded in the rheometer. The extension rate $\dot{\epsilon}$ was kept constant at $0.1 \mathrm{~s}^{-1}$ in all experiments. The maximum achievable strain with the EVF setup was limited to ca. 2.7, which is a too low strain value to reach failure in the unfermented dough samples prepared without yeast metabolites. The extensional viscosity curves are the average of at least 15 measurements on at least 3 separately prepared batches. As these measurements are not all independent, the pooled standard deviation $s_{p}$ has been used to determine the data variability (McNaught and Wilkinson, 1997):

$$
s_{p}=\sqrt{\frac{\sum_{i}\left[\left(n_{i}-1\right) s_{i}^{2}\right]}{\sum_{i}\left[n_{i}\right]-N}}
$$

In this expression, $s_{i}$ represents the standard deviation for the measurements of batch $\mathrm{i}$. The number of measurements in each batch is denoted by $n_{i}$, whereas the total number of batches is given by $N$. The relative standard deviation varied between 12 and $15 \%$ for unfermented dough without yeast metabolites. However, for unfermented dough with yeast metabolites and for fermented dough the data variability was much higher due 
to the premature failure of some of the samples. Consequently, for these materials a representative curve will be shown and not the average curve. For additional information on the rheological setups and methodologies the reader is referred to Meerts et al. (2017).

\section{Results and discussion}

\subsection{The rheological impact of yeast metabolites on the dough matrix in unfermented dough}

To elucidate the impact of the main yeast metabolites on the fundamental rheological properties of dough, adequate quantities of the metabolites were added directly to unfermented dough. Hereto, the metabolite concentrations as produced by yeast were determined by means of HPLC in fermented dough after 6 hours of fermentation, as described in section 2.3. The extended fermentation period of 6 hours was used to ensure that the yeast no longer showed significant activity during the rheological experiments (cf. section 3.2.1). Ethanol and glycerol reached concentrations of up to $90 \pm 0.54 \mathrm{mmol}$ and $8.8 \pm 0.01 \mathrm{mmol} / 100 \mathrm{~g}$ dry flour, respectively. The most abundant acid was succinic acid at $1.5 \pm 0.24 \mathrm{mmol} / 100 \mathrm{~g}$ dry flour, followed by acetic acid at $0.03 \pm 0.0001$ $\mathrm{mmol} / 100 \mathrm{~g}$ dry flour. The amount of lactic acid in the fermented dough was below the detection limit. Given the unusually long fermentation time used in this study (6 hours, whereas during breadmaking the fermentation phase typically lasts only for a few hours at most (Mondal and Datta, 2008)) and the rather high yeast concentration (5.3 wt\%, whereas a concentration of ca. 2 wt\% is more commonly applied (Mondal and Datta, 2008)), the metabolite concentrations reported here represent an upper limit for the concentrations that can be encountered in real-life breadmaking processes. The amount of glutathione in fermented dough was determined by Verheyen et al. (2015), and was found to vary considerably, also within different yeast batches of the same yeast type. To critically assess the potential of glutathione to change the rheological properties of the dough matrix, a representative concentration value of $0.03 \mathrm{mmol} / 100 \mathrm{~g}$ dry flour was used as determined by Verheyen et al. (2015) in their extensive survey of 27 commercial yeast types.

\subsubsection{Effect of ethanol}

The impact of ethanol on the linear and non-linear rheological properties of dough is shown in Fig. 1. The addition of ethanol at a concentration found in fermented dough (after prolonged fermentation) results in a substantial reduction of the dynamic moduli (two-tailed $p$-value $=0.002$ for $G^{\prime}(\omega=100 \mathrm{rad} / \mathrm{s})$ ). The extensional tests indicate that ethanol also lowers the extensional viscosity and failure strain of dough (at $\epsilon=2.2$ about half of the ethanol-supplemented dough samples had already experienced failure, whereas none of the reference dough samples had failed). In Kieffer rig extensibility tests, Jayaram et al. (2014a) equally observed a significant decrease in dough extensibility following the addition of ethanol. The effect of ethanol cannot merely be ascribed 
to an increase in solvent volume. When adding an equivalent volume of water to the dough instead of ethanol, the values of the dynamic moduli also decrease, but to a lesser extent (Fig. 1a). The difference in behaviour is even more apparent in the non-linear tests (Fig. 1b). The concurrent decline in dough stiffness, extensional viscosity and extensibility following the addition of ethanol indicates that the gluten network becomes less well-aggregated. The partial solubilisation of the gliadin fraction by the aqueous ethanol (which already starts at ca. 2 vol\% ethanol according to Jayaram et al. (2014a)) might indeed limit the number of non-covalent interactions within the gluten network, resulting in a more open and less cohesive network structure (Robertson et al., 1999). A more open network would also exhibit increased absorption capacity; as a matter of fact the extent of swelling of the gluten network in aqueous ethanol was found to correlate well with the solubility of the gliadin fraction, with both reaching a maximum at $50 \mathrm{vol} \%$ ethanol solution (Robertson et al., 1999). In the dough system under investigation, the ethanol concentration is far lower than this value, equalling about 6 vol $\%$ of the water fraction in dough.

\subsubsection{Effect of succinic acid}

Fig. 2 illustrates the impact of adding succinic acid on the linear and non-linear behaviour of wheat flour dough. The rheological behaviour of dough supplemented with succinic acid at the concentration observed in fermented dough $(1.5 \mathrm{mmol} / 100 \mathrm{~g}$ dry flour) turned out not to be significantly different from that of untreated dough (data not shown). Only at higher concentrations does succinic acid alter the rheological response. Following the addition of $4.5 \mathrm{mmol}$ succinic acid/100 g dry flour, the dynamic moduli decrease considerably (Fig. 2a). The extensional viscosity and extensibility are also negatively affected (Fig. $2 \mathrm{~b}$ ): at $\epsilon=2.6$ one-third of the dough samples containing succinic acid already experienced failure.

The rheological implications of adding acid to dough have already been studied extensively, but the results reported in literature are often contradictory. The disagreement stems from the complex interplay between the effect of $\mathrm{pH}$ and the effect of salt on the gluten network. Solvent retention tests (Jayaram et al., 2014b) and CLSM images (Schober et al., 2003) have revealed that, following the addition of acid, the flour proteins tend to swell and unfold. According to Wrigley (1968), the gluten proteins have an isoelectric point ranging from $\mathrm{pH} 6.0$ to $\mathrm{pH}$ 9.0. The $\mathrm{pH}$ of the unfermented dough used in this study equals $5.91 \pm 0.01$, which corresponds well with the $\mathrm{pH}$ values (about 5.8-6.0) typically reported in literature for unfermented dough (Jayaram et al., 2013; Verheyen et al., 2014). The addition of succinic acid results in a significant decrease in $\mathrm{pH}$ : the $\mathrm{pH}$ drops to $5.25 \pm 0.04$ for $1.5 \mathrm{mmol} / 100 \mathrm{~g}$ dry flour, and further down to $4.57 \pm 0.04$ for $4.5 \mathrm{mmol} / 100 \mathrm{~g}$ dry flour, thus coinciding with the $\mathrm{pH}$ range of 4.7-5.0 reported for fermented dough systems by Jayaram et al. (2013). This decrease in the $\mathrm{pH}$ resulting from the addition of acid thus leads to a net positive charge on the gluten proteins (due to the protonation of some of the carboxyl anions of the glutamic and aspartic acid residues). As a result, the gluten chains start to repel each other (Galal 
et al., 1978). Hence, upon lowering of the $\mathrm{pH}$, the gluten network typically experiences an overall loss of cohesiveness, resulting in smaller gluten aggregates (Jayaram et al., 2014b), lower values of the dynamic moduli (Thiele et al., 2003) and reduced extensibility and tensile strength (Harinder and Bains, 1990). In addition, lower $\mathrm{pH}$ values might prevent the formation of disulfide (SS) bonds during dough mixing. It is widely assumed that during dough mixing at ambient conditions, the gluten network is formed by an SH-SS interchange reaction mechanism, which requires free thiolate $\mathrm{S}^{-}$anions to carry out nucleophilic attacks on the sulfur atoms that are already engaged in pre-existing SS-bonds. The addition of acid will result in a lack of free $\mathrm{S}^{-}$anions, and will thus inhibit the SH-SS interchange reactions. Thiele et al. (2003) indeed report that at low pH (in casu ca. 3.5) the glutenin proteins have lost their ability to polymerise into glutenin macropolymer (GMP) during dough mixing. They additionally observed enhanced proteolytic breakdown of gluten by proteases endogenously present in wheat flour, as several of these enzymes have their maximum activity at lower $\mathrm{pH}$ levels (around 4.4, according to Kawamura and Yonezawa (1982)). These three factors combined may thus explain why low $\mathrm{pH}$ values typically result in a reduced gluten network strength.

However, the picture changes when salt is included in the dough recipe. As Galal et al. (1978) already pointed out, the salt ions have the ability to screen some, if not all, of the electrostatic repulsions, enabling the exposed hydrophobic groups to interact more intensively. CLSM imaging indeed showed that the gluten structure becomes increasingly dense upon the combined addition of both acid and salt (Schober et al., 2003). The addition of salt may thus (partially) reverse the detrimental effects of low $\mathrm{pH}$ values with regard to the gluten network cohesiveness; in some cases the rheological performance of dough supplemented with both acid and salt was found to be even superior to that of control dough, resulting in higher dynamic moduli (Thiele et al., 2003) and a higher tensile strength (Harinder and Bains, 1990). The extent to which salt can offset the adverse effects of low $\mathrm{pH}$ values is likely to depend on the $\mathrm{pH}$ level, salt concentration, and the inherent strength of the native gluten network. In the case of weak Bison flour, the weakening effect of the increased level of acidity clearly gained the upper hand at high succinic acid concentrations, whereas no significant effects could be observed for the succinic acid concentration level produced by yeast during fermentation.

Besides succinic acid, several other acids can be found in (yeast-fermented) wheat flour dough. However, their rheological impact will obviously be very similar to that of succinic acid. Furthermore, the concentration of the other acids in dough was found to be much lower than that of succinic acid, as discussed in the beginning of section 3.1.

\subsubsection{Effect of glycerol}

Despite its limited release in dough $(8.8 \mathrm{mmol} / 100 \mathrm{~g}$ dry flour $)$, glycerol was found to lower the dynamic moduli to a significant extent (Fig. 2a). The effect of glycerol is qualitatively similar to that of water, as both moduli tend to decrease whereas their frequency dependence remains unchanged (see also Zhou et al. (2016)). The softening 
effect of glycerol is also readily apparent in the extensional tests (Fig. 2b). As with the water content, the addition of glycerol leads to a downward vertical shift of the extensional viscosity curve, predominantly at small to moderate strains, whereas the viscosity increase at large strains is reduced less. The downward shift associated with the addition of glycerol is almost ten times larger than the shift obtained when adding an equivalent volume of water (results not shown). Besides a lower tensile strength, Aslankoohi et al. (2015) additionally observed an increase in the dough's extensibility in Kieffer rig tests using similar low levels of glycerol. They also found that glycerol led to an improvement of the dough's gas retention capacity during fermentation.

\subsubsection{Effect of glutathione}

Glutathione is a cell-protecting metabolite, produced by yeast when experiencing stressful conditions. Even though glutathione is normally not actively excreted by yeast, small amounts of glutathione have been identified in dough, as following cell death, the cell membranes will no longer prevent the cell contents from leaking to the environment (Verheyen et al., 2015). In contrast to the other main yeast metabolites (ethanol, succinic acid, glycerol), of which the concentrations were found to increase gradually over time (Jayaram et al., 2013), glutathione may be present in dough already at the beginning of fermentation (Verheyen et al., 2015). Once released, glutathione has the ability to cleave SS-bonds through the SH-SS interchange reaction mechanism mentioned earlier, which has a severe weakening effect on the dough (Dong and Hoseney, 1995).

To assess the impact of glutathione on the unfermented dough used in this study, a small amount of glutathione $(0.03 \mathrm{mmol} / 100 \mathrm{~g}$ dry flour $)$ representative of what has previously been detected in dough (Verheyen et al., 2015), was added to the flour just before mixing, and its rheological response after 6 hours was compared to that of unfermented control dough. Glutathione turned out to have a strong and lasting effect on both the dynamic moduli as well as the extensional response (Fig. 2). The dynamic moduli decreased considerably, with $G^{\prime}$ being affected the most, confirming the earlier findings of Dong and Hoseney (1995) and Verheyen et al. (2015). The resistance to extension also diminished, but failure could not be observed. Empirical extensional tests performed by Kieffer et al. (1990) equally showed that the addition of glutathione negatively affected the dough strength, whereas the extensibility improved slightly. Hence, our results indicate that glutathione, even at very low amounts, has a strong impact on dough rheology.

\subsection{The rheological behaviour of the dough matrix in fermented dough}

\subsubsection{Linear behaviour}

Obtaining non-evolving yeast-containing dough is not straightforward. Even after 6 hours of fermentation, the yeast still showed some residual activity (albeit very limited) 
that could be detected by the normal force transducer of the rheometer. The remaining yeast activity probably reflects the ability of amylases to degrade native starch (in addition to damaged starch), thus providing the yeast with a slow but steady influx of maltose. In addition, the sheeting action with the pasta machine is likely to have caused a redistribution of the available sugars within the dough, enabling the yeast to access new carbon sources. The use of even longer fermentation times ( 8 hours instead of 6 hours) did not suffice to suppress this residual activity (results not shown). However, the time-dependency of the dynamic moduli introduced by the ongoing fermentation proved to be very weak, as performing the entire frequency sweep from low to high frequency or high to low frequency did not significantly alter the rheological data (results not shown). Hence, we concluded that the time-dependency induced by the residual yeast activity could be safely ignored. As noted in a previous publication (Meerts et al., 2017), the dough should be given sufficient resting time after sheeting to allow the stresses induced by the sheeting action to relax. Time sweeps (angular frequency $\omega=1$ $\mathrm{rad} / \mathrm{s}$, strain amplitude $\gamma_{0}=0.06 \%$ ) on unfermented dough indicated that the resting period between sheeting and loading of the sample in the rheometer could be safely discarded, but that a resting time of at least 15 min should be observed after loading to obtain a quasi-steady-state response at the lowest frequency probed $(0.1 \mathrm{rad} / \mathrm{s})(\mathrm{results}$ not shown). Similar resting times were observed by Newberry et al. (2002) and Salvador et al. (2006). Hence, the frequency sweep was started after a time sweep of 5 min and was carried out from high to low frequency to guarantee a quasi-steady-state response at all frequencies probed. To allow a proper comparison, unfermented dough was subjected to exactly the same treatment as fermented dough.

Fig. 3a gives the dynamic moduli for both unfermented and fermented dough as a function of angular frequency. At low frequencies $G^{\prime}(\omega)$ of fermented dough drops significantly, indicating that the elastic response is severely weakened at long time scales. By contrast, at higher frequencies no significant difference could be observed between the moduli of unfermented and fermented dough. Whereas the impact of succinic acid was indeed found to be negligible at concentrations corresponding to those found in fermented dough (see section 3.1.2), the other metabolites did have a substantial effect, resulting in a global, parallel or non-parallel decrease in the moduli over the entire frequency range. The discrepancy in the rheological behaviour of fermented dough compared to that of unfermented dough supplemented with yeast metabolites could be caused by many factors. Repetitive frequency sweeps over the course of 2 hours on dough samples prepared with yeast and sheeted after a fermentation time of either 0 or 6 hours, indicated that the (re-)emergence of $\mathrm{CO}_{2}$ gas bubbles due to the ongoing fermentation process immediately resulted in a substantial drop in $G^{\prime}$ and $G^{\prime \prime}$ over the entire frequency range, as a result of the change in dough density. The data in Fig. 3a thus suggest that in our study the gas bubbles were removed adequately by the sheeting action, and hence did not cause the observed discrepancy. Another explanation could be that in fermented dough the simultaneous presence of the metabolites leads to specific interaction effects. Yet, the combined effect of adding the three main metabolites (ethanol, succinic acid, glycerol) simultaneously to unfermented dough did not differ 
significantly from the effect obtained when only ethanol is added (results not shown). A third possible explanation is that the changes observed in fermented dough reflect the time-dependent accumulation of the metabolites in the dough matrix. The gradual release of metabolites to a pre-existing gluten network might have less severe consequences as compared to the instant addition of significant amounts of metabolites to a dough system that still needs to be developed. However, contrary to ethanol, succinic acid and glycerol, some yeast metabolites (such as glutathione) are readily available and do exhibit immediate activity early on in the fermentation process (Verheyen et al., 2015). As a matter of fact, size-exclusion HPLC tests have revealed that in yeast-containing dough, a significant portion of the high molecular weight gluten molecules is broken down within only a few minutes after dough preparation (Newberry et al., 2002). The pronounced drop in the value of $G^{\prime}$ for fermented dough at low frequencies might well reflect this breakdown of the gluten network, as the longest material time scales in dough can be associated with the gluten proteins (Meerts et al., 2017). Glutathione is definitely able to produce such a dramatic change in the gluten molecular weight distribution within such a short time span, but other yeast components, such as yeast enzymes (Newberry et al., 2002), may equally be involved. The gluten proteins may also experience additional damage later on during fermentation as the gluten network is stretched biaxially by the expanding $\mathrm{CO}_{2}$ gas bubbles, which would result in a further loss of elasticity. Finally, it is interesting to note that some rheological studies on fermented dough using thermal inactivation did find $G^{\prime}$ and $G^{\prime \prime}$ to decrease as a consequence of fermentation, over a wide range of frequencies $(0.01-10 \mathrm{~Hz})$ (Salvador et al., 2006; Connelly and McIntier, 2008), whereas other studies did not observe any change (Newberry et al., 2002). As the very invasive thermal inactivation process effectively degassed the fermented dough samples (Newberry et al., 2002), these studies can also be trusted to solely probe the rheological changes related to the dough matrix, and not the changes associated with the presence of gas bubbles. The disparity in the findings of these studies on degassed fermented dough thus indicates that the rheological impact of the yeast metabolites on the dough matrix is not always clearly discernible in linear oscillatory tests.

\subsubsection{Non-linear behaviour}

Fig. 3b compares the extensional viscosity of fermented dough to that of unfermented dough. After sheeting of the dough sample, a resting time of $900 \mathrm{~s}$ was applied to guarantee good reproducibility in extensional tests on unfermented dough. Yet, for fermented dough most of the samples experienced failure before the EVF drum completed its turn, thus giving rise to much more data variability. Fig. 3b shows that following fermentation the dough's extensibility declines significantly. A decrease in extensibility might well reflect the action of (some of) the yeast metabolites, ethanol in particular (see section 3.1.1). However, at smaller strains the extensional viscosity of fermented dough is very similar to that of unfermented dough. As with the oscillatory tests, the extensional tests thus indicate that at small strains, the impact of the yeast metabolites is far less pronounced in fermented dough than in unfermented dough. The extensional tests additionally confirm that in fermented dough the gluten network suffered signif- 
icant damage, as indicated by the decrease in maximum extensional viscosity. It is important to note that any residual presence of gas bubbles could equally account for the detected changes in behaviour: also in active fermented dough, a decline in dough strength and extensibility has been observed (Kilborn and Preston, 1982). However, a decline in the dough's resistance against stretching and/or extensibility has also been reported for thermally inactivated fermented dough (Newberry et al., 2002), which probably no longer contained any gas bubbles due to the invasive nature of the inactivation method, and for active fermented dough from which the gas bubbles had been removed by sheeting (Rezaei et al., 2016). So the decrease in dough resistance and extensibility observed for fermented dough (Fig. 3b) most likely does not result from a residual presence of gas bubbles but from changes in the dough matrix brought about by yeast metabolites and/or by the expansion action itself (see section 3.2.1).

\section{Conclusions}

The main yeast metabolites were all found to have a clear softening effect on unfermented dough, even though their mechanisms were shown to be substantially different. Whereas glycerol merely had a diluting effect, ethanol, succinic acid and glutathione fundamentally altered the structure of the gluten network. However, in degassed fermented dough the softening effect was noticeable only at low frequencies (in oscillatory tests) or at high strains (in extensional tests). The rheological behaviour of degassed fermented dough can therefore not be explained simply as the superposition of the rheological effects of the individual yeast metabolites. During fermentation, the metabolites gradually accumulate in an already expanding gluten network, and this time-dependent accumulation is hard to simulate in unfermented dough. Additionally, yeast might release many other rheologically active components which were not covered in this study. Further research is therefore required to fully explain the rheological changes associated with yeast fermentation in terms of the chemical substances produced by yeast in dough.

\section{Acknowledgments}

MM is indebted to the Research Foundation - Flanders (FWO) for a doctoral fellowship at KU Leuven. The authors would also like to express their gratitude to the Research Fund KU Leuven (IDO/12/011) for financial support. Finally we would like to thank Mohammad Naser Rezaei for the fruitful discussions.

\section{References}

AOAC International, Official Methods of Analysis of AOAC International, 16th edition (AOAC International, Washington, DC, 1995) 
AACC International, Approved Methods of Analysis, 11th edition (AACC International, St. Paul, 2000)

Aslankoohi, E., Rezaei, M.N., Vervoort, Y., Courtin, C.M., Verstrepen, K.J., 2015. Glycerol production by fermenting yeast cells is essential for optimal bread dough fermentation. PLoS ONE 10, e0119364.

Connelly, R.K., McIntier, R.L., 2008. Rheological properties of yeasted and nonyeasted wheat doughs developed under different mixing conditions. Journal of the Science of Food and Agriculture 88, 2309-2323.

Dong, W., Hoseney, R.C., 1995. Effects of certain breadmaking oxidants and reducing agents on dough rheological properties. Cereal Chemistry 72, 58-64.

Galal, A.M., Varriano-Marston, E., Johnson, J.A., 1978. Rheological dough properties as affected by organic acids and salt. Cereal Chemistry 55, 683-691.

Harinder, K., Bains, G.S., 1990. High $\alpha$-amylase flours: effect of pH, acid, and salt on the rheological properties of dough. Cereal Chemistry 67, 588-594.

Jayaram, V.B., Cuyvers, S., Lagrain, B., Verstrepen, K.J., Delcour, J.A., Courtin, C.M., 2013. Mapping of Saccharomyces cerevisiae metabolites in fermenting wheat straightdough reveals succinic acid as pH-determining factor. Food Chemistry 136, 301-308.

Jayaram, V.B., Rezaei, M.N., Cuyvers, S., Verstrepen, K.J., Delcour, J.A., Courtin, C.M., 2014a. Ethanol at levels produced by Saccharomyces cerevisiae during wheat dough fermentation has a strong impact on dough properties. Journal of Agricultural and Food Chemistry 62, 9326-9335.

Jayaram, V.B., Cuyvers, S., Verstrepen, K.J., Delcour, J.A., Courtin, C.M., 2014b. Succinic acid in levels produced by yeast (Saccharomyces cerevisiae) during fermentation strongly impacts wheat bread dough properties. Food Chemistry 151, 421-428.

Kawamura, Y., Yonezawa, D., 1982. Wheat flour proteases and their action on gluten proteins in dilute acetic acid. Agricultural and Biological Chemistry 46, 767-773.

Kieffer, R., Kim, J.-J., Walther, C., Laskawy, G., Grosch, W., 1990. Influence of glutathione and cysteine on the improver effect of ascorbic acid stereoisomers. Journal of Cereal Science 11, 143-152.

Kilborn, R.H., Preston, K.R., 1982. A modified extensigraph procedure for measuring the stretching properties of fermented dough. Cereal Chemistry 59, 381-384.

McNaught, A.D., Wilkinson, A., Compendium of Chemical Terminology, 2nd edition (Blackwell Scientific Publications, Oxford, 1997)

Meerts, M., Cardinaels, R., Oosterlinck, F., Courtin, C. M., Moldenaers, P., 2017a. The interplay between the main flour constituents in the rheological behaviour of wheat flour dough. Food and Bioprocess Technology 10, 249-265. 
Mondal, A., Datta, A. K., 2008. Bread baking - a review. Journal of Food Engineering $86,465-474$.

Newberry, M.P., Phan-Thien, N., Larroque, O.R., Tanner, R.I., Larsen, N.G., 2002. Dynamic and elongation rheology of yeasted bread doughs. Cereal Chemistry 79, 874879.

Rezaei, M.N., Jayaram, V.B., Verstrepen, K.J., Courtin, C.M., 2016. The impact of yeast fermentation on dough matrix properties. Journal of the Science of Food and Agriculture 96, 3741-3748.

Robertson, G.H., Cao, T.K., Ong, I., 1999. Wheat gluten swelling and partial solubility with potential impact on starch-from-gluten separation by ethanol washing. Cereal Chemistry 76, 843-845.

Salvador, A., Sanz, T., Fiszman, S.M., 2006. Dynamic rheological characteristics of wheat flour-water doughs. Effect of adding $\mathrm{NaCl}$, sucrose and yeast. Food Hydrocolloids 20, 780-786.

Schober, T.J., Dockery, P., Arendt, E.K., 2003. Model studies for wheat sourdough systems using gluten, lactate buffer and sodium chloride. European Food Research and Technology 217, 235-243.

Thiele, C., Gänzle, M.G., Vogel, R.F. 2003. Fluorescence labeling of wheat proteins for determination of gluten hydrolysis and depolymerization during dough processing and sourdough fermentation. Journal of Agricultural and Food Chemistry 51, 2745-2752.

Verheyen, C., Jekle, M., Becker, T., 2014. Effects of Saccharomyces cerevisiae on the structural kinetics of wheat dough during fermentation. LWT - Food Science and Technology 58, 194-202.

Verheyen, C., Albrecht, A., Herrmann, J., Strobl, M., Jekle, M., Becker, T., 2015. The contribution of glutathione to the destabilizing effect of yeast on wheat dough. Food Chemistry 173, 243-249.

Wehrle, K., Arendt, E.K., 1998. Rheological changes in wheat sourdough during controlled and spontaneous fermentation. Cereal Chemistry 75, 882-886.

Wrigley, C.W., 1968. Gel electrofocusing: a technique for analyzing multiple protein samples by isoelectric focusing. Science Tools 15, 17-23.

Zhou, C.-F., Qian, P., Meng, J., Gao, S.-M., Lu, R.-R., 2016. Effect of glycerol and sorbitol on the properties of dough and white bread. Cereal Chemistry 93, 196-200. 


\section{List of Figures}

1 (a) Dynamic moduli $G^{\prime}(\omega)$ and $G^{\prime \prime}(\omega)$ and (b) extensional viscosity $\eta_{e}^{+}(\epsilon)$ at extension rate $\dot{\epsilon}=0.1 \mathrm{~s}^{-1}$ for unfermented dough and unfermented dough supplemented with $5.3 \mathrm{ml} / 100 \mathrm{~g}$ dry flour (=90 mmol/100 g dry flour) ethanol or $5.3 \mathrm{ml} / 100 \mathrm{~g}$ dry flour water. Dough samples were measured after a resting period of 6 hours in the climate chamber followed by an additional resting time of $300 \mathrm{~s}$ in the rheometer for (a) or an additional resting time of $900 \mathrm{~s}$ under a metal bowl for (b). The error bars shown in (a) at the lowest and highest frequency data points indicate the standard deviation. The depicted curves in (b) represent either the average (for the control dough and the unfermented dough supplemented with water) or the representative response (for the unfermented dough supplemented with ethanol). . . . . . . . . . . . . (a) Dynamic moduli $G^{\prime}(\omega)$ and $G^{\prime \prime}(\omega)$ and (b) extensional viscosity $\eta_{e}^{+}(\epsilon)$ at extension rate $\dot{\epsilon}=0.1 \mathrm{~s}^{-1}$ for unfermented dough and unfermented dough supplemented with $4.5 \mathrm{mmol} / 100 \mathrm{~g}$ dry flour succinic acid, 8.8 $\mathrm{mmol} / 100 \mathrm{~g}$ dry flour glycerol, or $0.03 \mathrm{mmol} / 100 \mathrm{~g}$ dry flour glutathione. Dough samples were measured after a resting period of 6 hours in the climate chamber followed by an additional resting time of $300 \mathrm{~s}$ in the rheometer for (a) or an additional resting time of $900 \mathrm{~s}$ under a metal bowl for (b). The error bars shown in (a) at the lowest and highest frequency data points indicate the standard deviation. The depicted curves in (b) represent either the average (for the control dough) or the representative response (for the unfermented dough supplemented with succinic acid, glycerol or glutathione). . . . . . . . . . . . . . . . .

3 (a) Storage modulus $G^{\prime}(\omega)$ and loss modulus $G^{\prime \prime}(\omega)$ and (b) extensional viscosity $\eta_{e}^{+}(\epsilon)$ at extension rate $\dot{\epsilon}=0.1 \mathrm{~s}^{-1}$ for unfermented dough and fermented dough without gas bubbles. Dough samples were measured after a fermentation period of 6 hours followed by an additional resting time of $300 \mathrm{~s}$ in the rheometer for (a) or an additional resting time of $900 \mathrm{~s}$ under a metal bowl for (b). The error bars shown in (a) at the lowest and highest frequency data points indicate the standard deviation. The depicted curves in (b) represent either the average (for unfermented dough) or the representative response (for fermented dough). . . . . . . . 


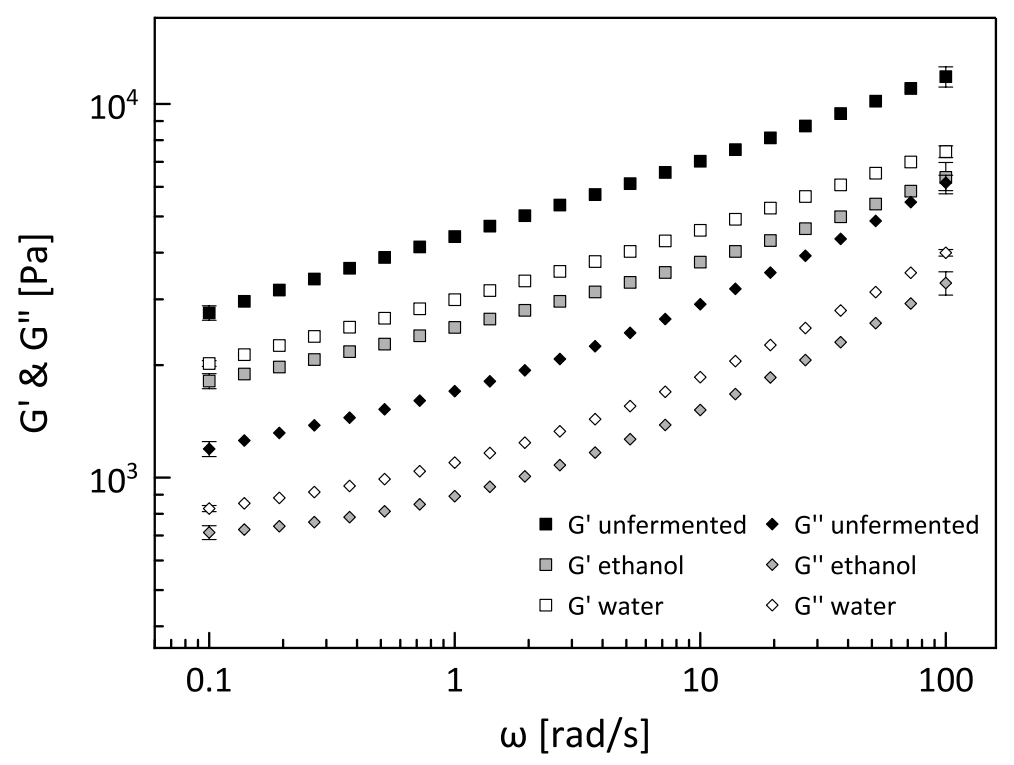

(a)

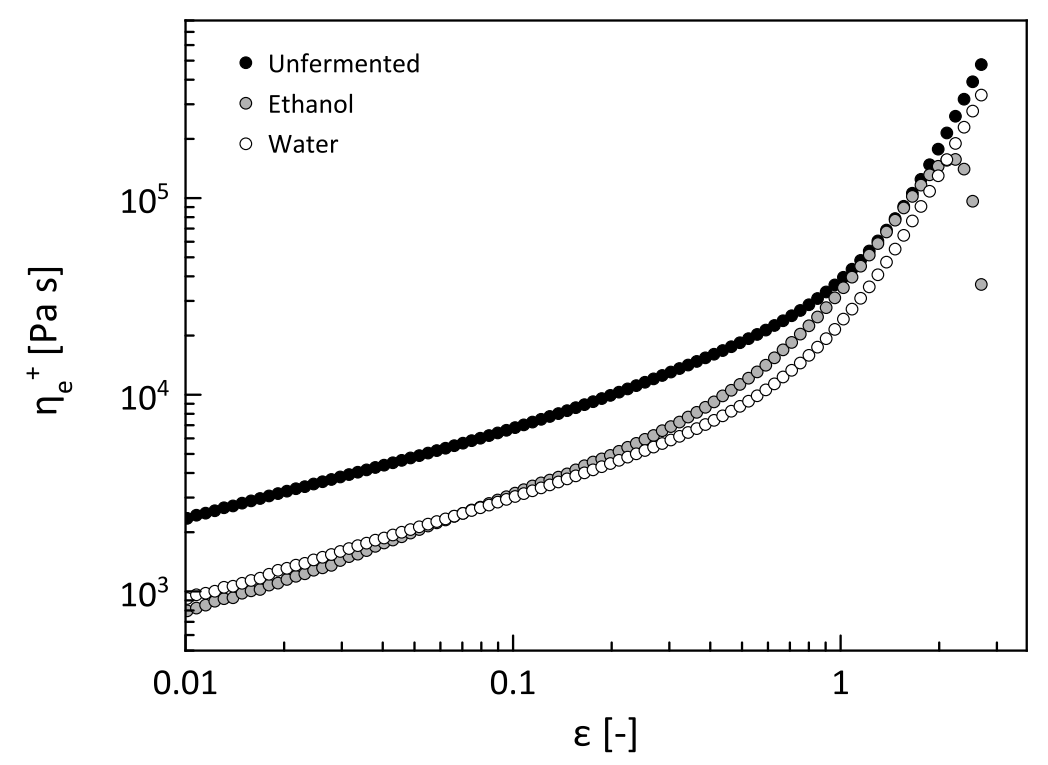

(b)

Figure 1: (a) Dynamic moduli $G^{\prime}(\omega)$ and $G^{\prime \prime}(\omega)$ and (b) extensional viscosity $\eta_{e}^{+}(\epsilon)$ at extension rate $\dot{\epsilon}=0.1 \mathrm{~s}^{-1}$ for unfermented dough and unfermented dough supplemented with $5.3 \mathrm{ml} / 100 \mathrm{~g}$ dry flour (=90 mmol/100 g dry flour) ethanol or $5.3 \mathrm{ml} / 100 \mathrm{~g}$ dry flour water. Dough samples were measured after a resting period of 6 hours in the climate chamber followed by an additional resting time of $300 \mathrm{~s}$ in the rheometer for (a) or an additional resting time of $900 \mathrm{~s}$ under a metal bowl for (b). The error bars shown in (a) at the lowest and highest frequency data points indicate the standard deviation. The depicted curves in (b) represent either the average (for the control dough and the unfermented dough supplemented with water) or the representative response (for the unfermented dough supplemented with ethanol). 


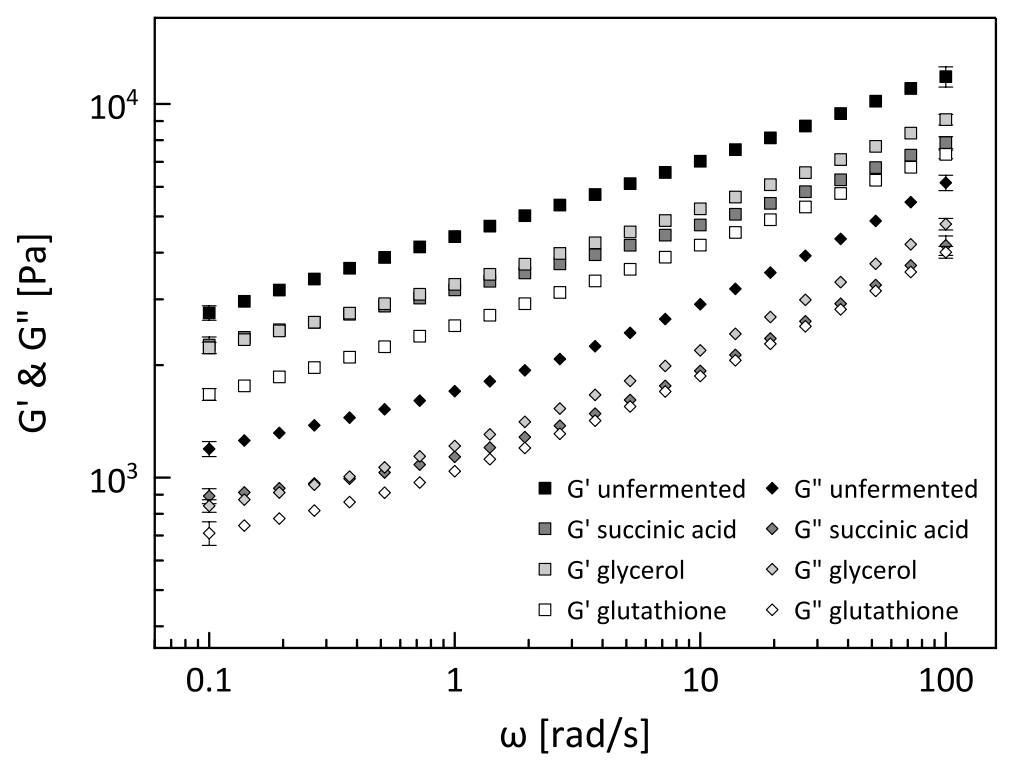

(a)

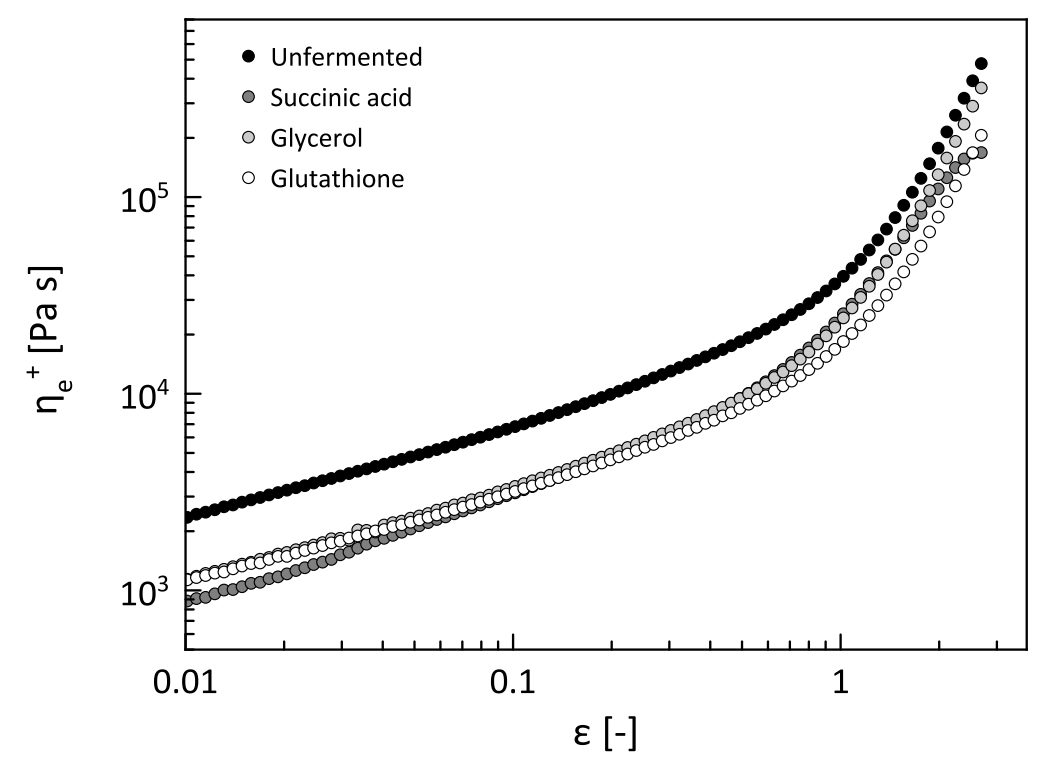

(b)

Figure 2: (a) Dynamic moduli $G^{\prime}(\omega)$ and $G^{\prime \prime}(\omega)$ and (b) extensional viscosity $\eta_{e}^{+}(\epsilon)$ at extension rate $\dot{\epsilon}=0.1 \mathrm{~s}^{-1}$ for unfermented dough and unfermented dough supplemented with $4.5 \mathrm{mmol} / 100 \mathrm{~g}$ dry flour succinic acid, $8.8 \mathrm{mmol} / 100 \mathrm{~g}$ dry flour glycerol, or 0.03 $\mathrm{mmol} / 100 \mathrm{~g}$ dry flour glutathione. Dough samples were measured after a resting period of 6 hours in the climate chamber followed by an additional resting time of $300 \mathrm{~s}$ in the rheometer for (a) or an additional resting time of $900 \mathrm{~s}$ under a metal bowl for (b). The error bars shown in (a) at the lowest and highest frequency data points indicate the standard deviation. The depicted curves in (b) represent either the average (for the control dough) or the representative response (for the unfermented dough supplemented with succinic acid, glycerol or glutathione). 


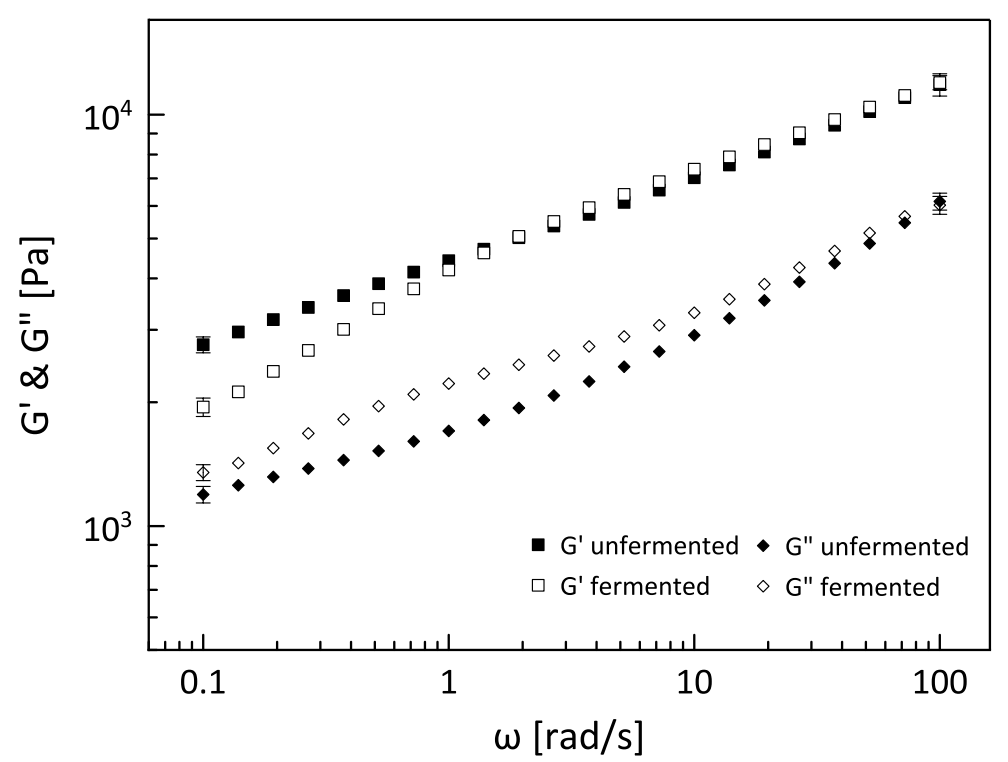

(a)

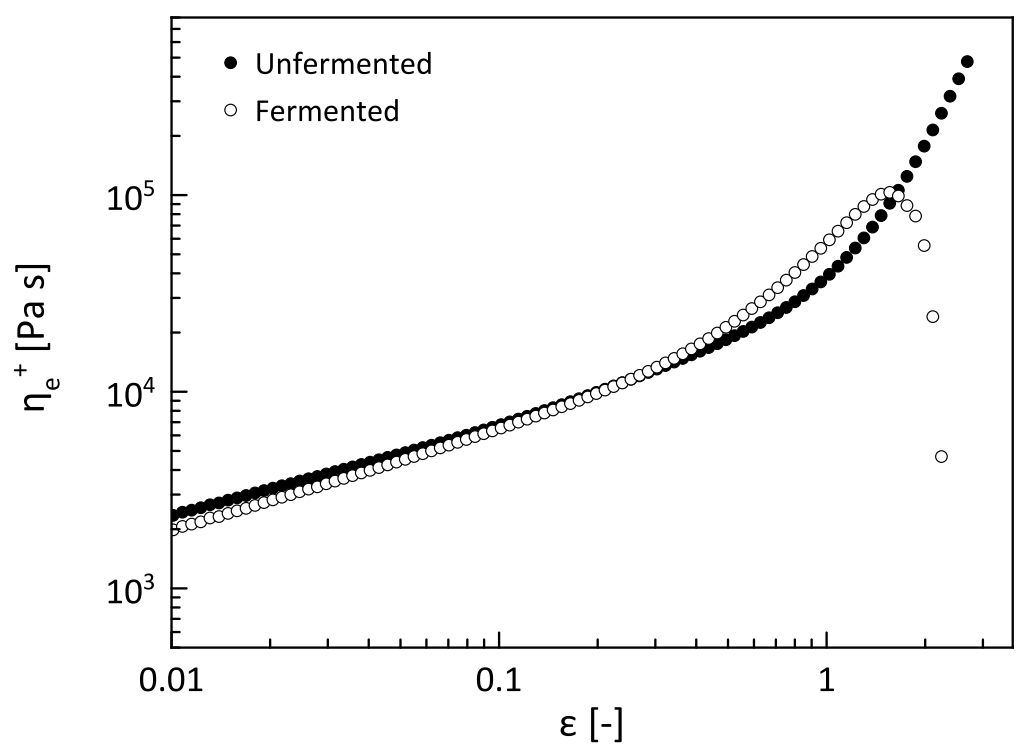

(b)

Figure 3: (a) Storage modulus $G^{\prime}(\omega)$ and loss modulus $G^{\prime \prime}(\omega)$ and (b) extensional viscosity $\eta_{e}^{+}(\epsilon)$ at extension rate $\dot{\epsilon}=0.1 \mathrm{~s}^{-1}$ for unfermented dough and fermented dough without gas bubbles. Dough samples were measured after a fermentation period of 6 hours followed by an additional resting time of $300 \mathrm{~s}$ in the rheometer for (a) or an additional resting time of $900 \mathrm{~s}$ under a metal bowl for (b). The error bars shown in (a) at the lowest and highest frequency data points indicate the standard deviation. The depicted curves in (b) represent either the average (for unfermented dough) or the representative response (for fermented dough). 